\title{
Quantum decoherence and classical correlations of the harmonic oscillator in the Lindblad theory
}

\author{
A. Isar ${ }^{1,2,(a)}$ and W. Scheid ${ }^{2}$ \\ ${ }^{1}$ Department of Theoretical Physics, Institute of Physics and Nuclear Engineering, \\ Bucharest-Magurele, Romania \\ 2 Institut für Theoretische Physik der Justus-Liebig-Universität, \\ Giessen, Germany
}

\begin{abstract}
In the framework of the Lindblad theory for open quantum systems we determine the degree of quantum decoherence and classical correlations of a harmonic oscillator interacting with a thermal bath. The transition from quantum to classical behaviour of the considered system is analyzed and it is shown that the classicality takes place during a finite interval of time. We calculate also the decoherence time and show that it has the same scale as the time after which statistical fluctuations become comparable with quantum fluctuations.
\end{abstract}

PACS numbers: 03.65.Yz, 05.30.-d

(a) e-mail address: isar@theory.nipne.ro

\section{Introduction}

The transition from quantum to classical physics and classicality of quantum systems continue to be among the most interesting problems in many fields of physics, for both conceptual and experimental reasons [1, 2, 3]. Two conditions are essential for the classicality of a quantum system [4, 5]: a) quantum decoherence (QD), that means the irreversible, uncontrollable and persistent formation of a quantum correlation (entanglement) of the system with its environment [6], expressed by the damping of the coherences present in the quantum state of the system, when the off-diagonal elements of the density matrix of the system decay below a certain level, so that this density matrix becomes approximately diagonal and b) classical correlations (CC), expressed by the fact that the Wigner function of the quantum system has a peak which follows the classical equations of motion in phase space with a good degree of approximation, that is the quantum state becomes peaked along a classical trajectory. The necessity and sufficiency of both QD and $\mathrm{CC}$ as conditions of classicality are still a subject of 
debate. Both these conditions do not have an universal character, so that they are not necessary for all physical models. An important role in this discussion plays the temperature of the environment and therefore it is worth to take into account the differences between low and high temperature regimes. For example, purely classical systems at very high temperatures are described by a classical Fokker-Planck equation which does not follow any trajectory in phase space (for very small kinetic energy, compared to the thermal energy, when the probability distribution becomes essentially independent of momentum), so that in this case CC are not necessary. Likewise, one can have a classical behaviour if the coherences are negligible, without having strong CC (for example, in the case of a classical gas at finite temperature) and the lack of strong correlations between the coordinate and its canonical momentum does not necessarily mean that the system is quantum. On the other hand, the condition of CC is not sufficient for a system to become classical - although the Wigner function can show a sharp correlation in phase space, the quantum coherence never vanishes for a closed system which has a unitary evolution. Likewise, in the low temperature quantum regime one can observe strong CC. For example, in the case of a purely damped quantum harmonic oscillator (at zero temperature), the initial coherent states remain coherent and perfectly follow classical trajectories of a damped oscillator, but CC are not sufficient for classicality.

In the last two decades it has became more and more clear that the classicality is an emergent property of open quantum systems, since both main features of this process - QD and CC - strongly depend on the interaction between the system and its external environment [3, 7, 8, 9, 10, 11, 12, 13, 14, 15]. A remarkable aspect of the current research helping in understanding the nature of the quantum to classical transition is that for the first time there have recently been carried on experiments probing the boundary between the quantum and the classical domains in a controlled way [16, 17, 18, 19, 20, 21].

The role of QD became relevant in many interesting physical problems of field theory, atomic physics, quantum optics, quantum information processing, quantum gravity and cosmology, and condensed matter physics. We mention here only a few of these problems [2, 3]: to understand the way in which QD favorizes the quantum to classical transition of density fluctuations; to study systems of trapped and cold atoms (or ions) which may offer the possibility of engineering the environment, like trapped atoms inside cavities, relation between decoherence and other cavity QED effects (such as Casimir effect); on mesoscopic scale, decoherence in the context of Bose-Einstein condensation.

In many cases one is interested in understanding the specific causes of QD just 
because one wants to prevent decoherence from damaging quantum states and to protect the information stored in quantum states from the degrading effect of the interaction with the environment. Thus, decoherence is responsible for washing out the quantum interference effects which are desirable to be seen as signals in some experiments. QD has a negative influence on many areas relying upon quantum coherence effects, such as quantum computation and quantum control of atomic and molecular processes. In the physics of information and computation, decoherence is an obvious major problem in the implementation of information-processing hardware that takes advantage of the superposition principle [22].

In most of literature, QD has been studied for a system coupled to an environment or thermal bath with many degrees of freedom. The main purpose of this paper is to study QD and CC for a harmonic oscillator interacting with an environment in the framework of the Lindblad theory for open quantum systems. More concretely we determine the degree of $\mathrm{QD}$ and $\mathrm{CC}$ and the possibility of simultaneous realization of $\mathrm{QD}$ and $\mathrm{CC}$ for a system consisting of a harmonic oscillator in a thermal bath. For that purpose, we first find the evolution of the density matrix and of the Wigner function of the considered system and then we apply the criterion of QD and CC. We consider different regimes of the temperature of environment. It is found that the system manifests a QD which increases with time and temperature, whereas CC are less and less strong with increasing time and temperature.

The organizing of the paper is as follows. In Sec. 2 we review the Lindblad master equation for the damped harmonic oscillator and in Sec. 3 we derive the master equation in coordinate representation and the corresponding Fokker-Planck equation in the Wigner representation and determine the density matrix and Wigner function of the considered system. Then in Sec. 4 we investigate QD and CC and analyze them quantitatively. In Sec. 5 we calculate the decoherence time of the system and discuss the transition from quantum mechanics to classical statistical mechanics. A summary and concluding remarks are given in Sec. 6.

\section{Lindblad master equation for the harmonic oscil- lator}

Here we review the Lindblad's axiomatic formalism based on quantum dynamical semigroups. The irreversible time evolution of an open system is described by the following 
general quantum Markovian master equation for the density operator $\rho(t)$ [23, 24, 25]:

$$
\frac{d \rho(t)}{d t}=-\frac{i}{\hbar}[H, \rho(t)]+\frac{1}{2 \hbar} \sum_{j}\left(\left[V_{j} \rho(t), V_{j}^{\dagger}\right]+\left[V_{j}, \rho(t) V_{j}^{\dagger}\right]\right) .
$$

$H$ is the Hamiltonian of the system and $V_{j}, V_{j}^{\dagger}$ are operators on the Hilbert space of $H$, which model the environment. In order to obtain, for the damped quantum harmonic oscillator, equations of motion as close as possible to the classical ones, the two possible operators $V_{1}$ and $V_{2}$ are taken as linear polynomials in coordinate $q$ and momentum $p$ [26, 27, 28] and the harmonic oscillator Hamiltonian $H$ is chosen of the general quadratic form

$$
H=H_{0}+\frac{\mu}{2}(q p+p q), \quad H_{0}=\frac{1}{2 m} p^{2}+\frac{m \omega^{2}}{2} q^{2} .
$$

With these choices the master equation (11) takes the following form [27, 28]:

$$
\begin{aligned}
\frac{d \rho}{d t} & =-\frac{i}{\hbar}\left[H_{0}, \rho\right]-\frac{i}{2 \hbar}(\lambda+\mu)[q, \rho p+p \rho]+\frac{i}{2 \hbar}(\lambda-\mu)[p, \rho q+q \rho] \\
& -\frac{D_{p p}}{\hbar^{2}}[q,[q, \rho]]-\frac{D_{q q}}{\hbar^{2}}[p,[p, \rho]]+\frac{D_{p q}}{\hbar^{2}}([q,[p, \rho]]+[p,[q, \rho]]) .
\end{aligned}
$$

The quantum diffusion coefficients $D_{p p}, D_{q q}, D_{p q}$ and the dissipation constant $\lambda$ satisfy the following fundamental constraints [27, 28]: $D_{p p}>0, D_{q q}>0$ and

$$
D_{p p} D_{q q}-D_{p q}^{2} \geq \frac{\lambda^{2} \hbar^{2}}{4} .
$$

In the particular case when the asymptotic state is a Gibbs state $\rho_{G}(\infty)=e^{-\frac{H_{0}}{k T}} / \operatorname{Tr} e^{-\frac{H_{0}}{k T}}$, these coefficients become [27, 28]

$$
D_{p p}=\frac{\lambda+\mu}{2} \hbar m \omega \operatorname{coth} \frac{\hbar \omega}{2 k T}, \quad D_{q q}=\frac{\lambda-\mu}{2} \frac{\hbar}{m \omega} \operatorname{coth} \frac{\hbar \omega}{2 k T}, \quad D_{p q}=0,
$$

where $T$ is the temperature of the thermal bath. In this case, the fundamental constraints are satisfied only if $\lambda>\mu$ and

$$
\left(\lambda^{2}-\mu^{2}\right) \operatorname{coth}^{2} \frac{\hbar \omega}{2 k T} \geq \lambda^{2} .
$$

From the master equation (3) we obtain the following equations of motion for the expectation values of coordinate and momentum [27, 28]:

$$
\begin{gathered}
\frac{d \sigma_{q}(t)}{d t}=-(\lambda-\mu) \sigma_{q}(t)+\frac{1}{m} \sigma_{p}(t), \\
\frac{d \sigma_{p}(t)}{d t}=-m \omega^{2} \sigma_{q}(t)-(\lambda+\mu) \sigma_{p}(t) .
\end{gathered}
$$


In the underdamped case $(\omega>\mu)$ considered in this paper, with the notation $\Omega^{2} \equiv$ $\omega^{2}-\mu^{2}$, we obtain [27, 28]:

$$
\begin{gathered}
\sigma_{q}(t)=e^{-\lambda t}\left(\left(\cos \Omega t+\frac{\mu}{\Omega} \sin \Omega t\right) \sigma_{q}(0)+\frac{1}{m \Omega} \sin \Omega t \sigma_{p}(0)\right), \\
\sigma_{p}(t)=e^{-\lambda t}\left(-\frac{m \omega^{2}}{\Omega} \sin \Omega t \sigma_{q}(0)+\left(\cos \Omega t-\frac{\mu}{\Omega} \sin \Omega t\right) \sigma_{p}(0)\right)
\end{gathered}
$$

and $\sigma_{q}(\infty)=\sigma_{p}(\infty)=0$.

Lindblad has proven [26] that in the Markovian regime the harmonic oscillator master equation which satisfies the complete positivity condition cannot satisfy simultaneously the translational invariance and the detailed balance (which assures an asymptotic approach to the canonical thermal equilibrium state). The necessary and sufficient condition for translational invariance is $\lambda=\mu[26,27,28]$. In this case the equations of motion (77) and (88) are exactly the same as the classical ones. If $\lambda \neq \mu$, then we violate translational invariance, but we keep the canonical equilibrium state.

The relation (4) is a necessary condition for the generalized uncertainty inequality

$$
\sigma_{q q}(t) \sigma_{p p}(t)-\sigma_{p q}^{2}(t) \geq \frac{\hbar^{2}}{4}
$$

to be fulfilled, where $\sigma_{q q}$ and $\sigma_{p p}$ denote the dispersion (variance) of the coordinate and momentum, respectively, and $\sigma_{p q}$ denotes the correlation (covariance) of the coordinate and momentum. The equality in relation (11) is realized for a special class of pure states, called correlated coherent states [29] or squeezed coherent states.

The asymptotic values $\sigma_{q q}(\infty), \sigma_{p p}(\infty), \sigma_{p q}(\infty)$ do not depend on the initial values $\sigma_{q q}(0), \sigma_{p p}(0), \sigma_{p q}(0)$ and in the case of a thermal bath with coefficients (5), they reduce to [27, 28]

$$
\sigma_{q q}(\infty)=\frac{\hbar}{2 m \omega} \operatorname{coth} \frac{\hbar \omega}{2 k T}, \quad \sigma_{p p}(\infty)=\frac{\hbar m \omega}{2} \operatorname{coth} \frac{\hbar \omega}{2 k T}, \quad \sigma_{p q}(\infty)=0 .
$$

In the following, we consider a general temperature $T$, but we should stress that the Lindblad theory is obtained in the Markov approximation, which holds for high temperatures of the environment. At the same time, the semigroup dynamics of the density operator which must hold for a quantum Markovian process is valid only for the weak-coupling regime, with the damping $\lambda$ obeying the inequality $\lambda \ll \omega$.

\section{Density matrix and Wigner distribution function}

We consider a harmonic oscillator with an initial Gaussian wave function

$$
\Psi(q)=\left(\frac{1}{2 \pi \sigma_{q q}(0)}\right)^{\frac{1}{4}} \exp \left[-\frac{1}{4 \sigma_{q q}(0)}\left(1-\frac{2 i}{\hbar} \sigma_{p q}(0)\right)\left(q-\sigma_{q}(0)\right)^{2}+\frac{i}{\hbar} \sigma_{p}(0) q\right],
$$


where $\sigma_{q q}(0)$ is the initial spread, $\sigma_{p q}(0)$ the initial covariance, and $\sigma_{q}(0)$ and $\sigma_{p}(0)$ are the initial averaged position and momentum of the wave packet. The initial state (13) represents a correlated coherent state [29] with the variances and covariance of coordinate and momentum

$$
\sigma_{q q}(0)=\frac{\hbar \delta}{2 m \omega}, \quad \sigma_{p p}(0)=\frac{\hbar m \omega}{2 \delta\left(1-r^{2}\right)}, \quad \sigma_{p q}(0)=\frac{\hbar r}{2 \sqrt{1-r^{2}}} .
$$

Here, $\delta$ is the squeezing parameter which measures the spread in the initial Gaussian packet and $r$, with $|r|<1$ is the correlation coefficient at time $t=0$. The initial values (14) correspond to a minimum uncertainty state, since they fulfil the generalized uncertainty relation

$$
\sigma_{q q}(0) \sigma_{p p}(0)-\sigma_{p q}^{2}(0)=\frac{\hbar^{2}}{4}
$$

For $\delta=1$ and $r=0$ the correlated coherent state becomes a Glauber coherent state. For a given temperature $T$ of the bath and for any parameters $\delta$ and $r$ the inequality (6) alone determines the range of values of the parameters $\lambda$ and $\mu$ [30].

From Eq. (3) we derive the evolution equation in coordinate representation:

$$
\begin{array}{r}
\frac{\partial \rho}{\partial t}=\frac{i \hbar}{2 m}\left(\frac{\partial^{2}}{\partial q^{2}}-\frac{\partial^{2}}{\partial q^{\prime 2}}\right) \rho-\frac{i m \omega^{2}}{2 \hbar}\left(q^{2}-q^{2}\right) \rho \\
-\frac{1}{2}(\lambda+\mu)\left(q-q^{\prime}\right)\left(\frac{\partial}{\partial q}-\frac{\partial}{\partial q^{\prime}}\right) \rho+\frac{1}{2}(\lambda-\mu)\left[\left(q+q^{\prime}\right)\left(\frac{\partial}{\partial q}+\frac{\partial}{\partial q^{\prime}}\right)+2\right] \rho \\
-\frac{D_{p p}}{\hbar^{2}}\left(q-q^{\prime}\right)^{2} \rho+D_{q q}\left(\frac{\partial}{\partial q}+\frac{\partial}{\partial q^{\prime}}\right)^{2} \rho-2 i D_{p q} \hbar\left(q-q^{\prime}\right)\left(\frac{\partial}{\partial q}+\frac{\partial}{\partial q^{\prime}}\right) \rho
\end{array}
$$

and in Refs. [31, 32, 33] we transformed the master equation (3) for the density operator into the following Fokker-Planck-type equation satisfied by the Wigner distribution function $W(q, p, t)$ :

$$
\begin{aligned}
\frac{\partial W}{\partial t}=-\frac{p}{m} \frac{\partial W}{\partial q} & +m \omega^{2} q \frac{\partial W}{\partial p}+(\lambda+\mu) \frac{\partial}{\partial p}(p W)+(\lambda-\mu) \frac{\partial}{\partial q}(q W) \\
& +D_{p p} \frac{\partial^{2} W}{\partial p^{2}}+D_{q q} \frac{\partial^{2} W}{\partial q^{2}}+2 D_{p q} \frac{\partial^{2} W}{\partial p \partial q}
\end{aligned}
$$

The first two terms on the right-hand side of both these equations generate a purely unitary evolution. They give the usual Liouvillian evolution. The third and forth terms are the dissipative terms and have a damping effect (exchange of energy with environment). The last three are noise (diffusive) terms and produce fluctuation effects in the evolution of the system. $D_{p p}$ promotes diffusion in momentum and generates decoherence in coordinate $q$ : it reduces the off-diagonal terms, responsible for correlations 
between spatially separated pieces of the wave packet. Similarly $D_{q q}$ promotes diffusion in coordinate and generates decoherence in momentum $p$. The $D_{p q}$ term is the so-called "anomalous diffusion" term. It promotes diffusion in the variable $q p+p q$, just like both the other diffusion terms, but it does not generate decoherence.

In the high temperature limit, quantum Fokker-Planck equation (17) with coefficients (5) becomes classical Kramers equation $\left(D_{p p} \rightarrow 2 m \lambda k T\right.$ for $\left.\lambda=\mu\right)$ [33].

The density matrix solution of Eq. (16) has the general form of Gaussian density matrices

$$
\begin{array}{r}
<q|\rho(t)| q^{\prime}>=\left(\frac{1}{2 \pi \sigma_{q q}(t)}\right)^{\frac{1}{2}} \exp \left[-\frac{1}{2 \sigma_{q q}(t)}\left(\frac{q+q^{\prime}}{2}-\sigma_{q}(t)\right)^{2}\right. \\
\left.-\frac{\sigma(t)}{2 \hbar^{2} \sigma_{q q}(t)}\left(q-q^{\prime}\right)^{2}+\frac{i \sigma_{p q}(t)}{\hbar \sigma_{q q}(t)}\left(\frac{q+q^{\prime}}{2}-\sigma_{q}(t)\right)\left(q-q^{\prime}\right)+\frac{i}{\hbar} \sigma_{p}(t)\left(q-q^{\prime}\right)\right]
\end{array}
$$

where

$$
\sigma(t) \equiv \sigma_{q q}(t) \sigma_{p p}(t)-\sigma_{p q}^{2}(t)
$$

is the determinant of the dispersion (correlation) matrix

$$
\left(\begin{array}{cc}
\sigma_{q q}(t) & \sigma_{p q}(t) \\
\sigma_{p q}(t) & \sigma_{p p}(t)
\end{array}\right)
$$

and represents also the Schrödinger generalized uncertainty function [30].

For an initial Gaussian Wigner function (corresponding to a correlated coherent state (13)) the solution of Eq. (17) is

$$
\begin{array}{r}
W(q, p, t)=\frac{1}{2 \pi \sqrt{\sigma(t)}} \exp \left\{-\frac{1}{2 \sigma(t)}\left[\sigma_{p p}(t)\left(q-\sigma_{q}(t)\right)^{2}+\sigma_{q q}(t)\left(p-\sigma_{p}(t)\right)^{2}\right.\right. \\
\left.\left.-2 \sigma_{p q}(t)\left(q-\sigma_{q}(t)\right)\left(p-\sigma_{p}(t)\right)\right]\right\} .
\end{array}
$$

In the case of a thermal bath we obtain the following steady state solution for $t \rightarrow \infty$ (we denote $\left.\epsilon \equiv \frac{\hbar \omega}{2 k T}\right)$ :

$$
<q|\rho(\infty)| q^{\prime}>=\left(\frac{m \omega}{\pi \hbar \operatorname{coth} \epsilon}\right)^{\frac{1}{2}} \exp \left\{-\frac{m \omega}{4 \hbar}\left[\frac{\left(q+q^{\prime}\right)^{2}}{\operatorname{coth} \epsilon}+\left(q-q^{\prime}\right)^{2} \operatorname{coth} \epsilon\right]\right\} .
$$

In the long time limit we have also

$$
W_{\infty}(q, p)=\frac{1}{\pi \hbar \operatorname{coth} \epsilon} \exp \left\{-\frac{1}{\hbar \operatorname{coth} \epsilon}\left[m \omega q^{2}+\frac{p^{2}}{m \omega}\right]\right\} .
$$

Stationary solutions to the evolution equations obtained in the long time limit are possible as a result of a balance between the wave packet spreading induced by the Hamiltonian and the localizing effect of the Lindblad operators. 


\section{Quantum decoherence and classical correlations}

As we stated in the Introduction, one considers that two conditions have to be satisfied in order that a system could be considered as classical. The first condition requires that the system should be in one of relatively permanent states (states that are least affected by the interaction of the system with the environment, called by Zurek "preferred states" in the environment induced superselection description [2, 3]) and the interference between different states should be negligible. This implies the destruction of off-diagonal elements representing coherences between quantum states in the density matrix, which is the QD phenomenon. An isolated system has an unitary evolution and the coherence of the state is not lost - pure states evolve in time only to pure states. The loss of coherence can be achieved by introducing an interaction between the system and environment: an initial pure state with a density matrix which contains nonzero off-diagonal terms can non-unitarily evolve into a final mixed state with a diagonal density matrix during the interaction with the environment, like in classical statistical mechanics.

The second condition requires that the system should have, with a good approximation, an evolution according to classical laws. This implies that the Wigner distribution function has a peak along a classical trajectory, that means there exist CC between the canonical variables of coordinate and momentum. Of course, the correlation between the canonical variables, necessary to obtain a classical limit, should not violate Heisenberg uncertainty principle, i.e. the position and momentum should take reasonably sharp values, to a degree in concordance with the uncertainty principle. This is possible, because the density matrix does not diagonalize exactly in position, but with a non-zero width, i.e. it is strongly peaked about $q=q^{\prime}$ and very small for $q$ far from $q^{\prime}$.

Using new variables $\Sigma=\left(q+q^{\prime}\right) / 2$ and $\Delta=q-q^{\prime}$, the density matrix (18) can be rewritten as

$\rho(\Sigma, \Delta, t)=\sqrt{\frac{\alpha}{\pi}} \exp \left[-\alpha \Sigma^{2}-\gamma \Delta^{2}+i \beta \Sigma \Delta+2 \alpha \sigma_{q}(t) \Sigma+i\left(\frac{\sigma_{p}(t)}{\hbar}-\beta \sigma_{q}(t)\right) \Delta-\alpha \sigma_{q}^{2}(t)\right]$,

with the abbreviations

$$
\alpha=\frac{1}{2 \sigma_{q q}(t)}, \quad \gamma=\frac{\sigma(t)}{2 \hbar^{2} \sigma_{q q}(t)}, \quad \beta=\frac{\sigma_{p q}(t)}{\hbar \sigma_{q q}(t)}
$$

and the Wigner transform of the density matrix (24) is

$$
W(q, p, t)=\frac{1}{2 \pi \hbar} \sqrt{\frac{\alpha}{\gamma}} \exp \left\{-\frac{\left[\hbar \beta\left(q-\sigma_{q}(t)\right)-\left(p-\sigma_{p}(t)\right)\right]^{2}}{4 \hbar^{2} \gamma}-\alpha\left(q-\sigma_{q}(t)\right)^{2}\right\} .
$$




\section{a) Degree of quantum decoherence ( $Q D$}

The representation-independent measure of the degree of QD [4] is given by the ratio of the dispersion $1 / \sqrt{2 \gamma}$ of the off-diagonal element $\rho(0, \Delta, t)$ to the dispersion $\sqrt{2 / \alpha}$ of the diagonal element $\rho(\Sigma, 0, t)$ :

$$
\delta_{Q D}=\frac{1}{2} \sqrt{\frac{\alpha}{\gamma}},
$$

which in our case gives

$$
\delta_{Q D}(t)=\frac{\hbar}{2 \sqrt{\sigma(t)}} .
$$

The finite temperature Schrödinger generalized uncertainty function (19), calculated in Ref. [30], has the expression

$$
\begin{array}{r}
\sigma(t)=\frac{\hbar^{2}}{4}\left\{e^{-4 \lambda t}\left[1-\left(\delta+\frac{1}{\delta\left(1-r^{2}\right)}\right) \operatorname{coth} \epsilon+\operatorname{coth}^{2} \epsilon\right]\right. \\
+e^{-2 \lambda t} \operatorname{coth} \epsilon\left[\left(\delta+\frac{1}{\delta\left(1-r^{2}\right)}-2 \operatorname{coth} \epsilon\right) \frac{\omega^{2}-\mu^{2} \cos (2 \Omega t)}{\Omega^{2}}\right. \\
\left.\left.+\left(\delta-\frac{1}{\delta\left(1-r^{2}\right)}\right) \frac{\mu \sin (2 \Omega t)}{\Omega}+\frac{2 r \mu \omega(1-\cos (2 \Omega t))}{\Omega^{2} \sqrt{1-r^{2}}}\right]+\operatorname{coth}^{2} \epsilon\right\} .
\end{array}
$$

In the limit of long times Eq. (29) yields

$$
\sigma(\infty)=\frac{\hbar^{2}}{4} \operatorname{coth}^{2} \epsilon
$$

so that we obtain

$$
\delta_{Q D}(\infty)=\tanh \frac{\hbar \omega}{2 k T}
$$

which for high $T$ becomes

$$
\delta_{Q D}(\infty)=\frac{\hbar \omega}{2 k T}
$$

We see that $\delta_{Q D}$ decreases, and therefore QD increases, with temperature, i.e. the density matrix becomes more and more diagonal at higher $T$ and the contributions of the off-diagonal elements get smaller and smaller. At the same time the degree of purity decreases and the degree of mixedness increases with $T$. $\delta_{Q D}<1$ for $T \neq 0$, while for $T=0$ the asymptotic (final) state is pure and $\delta_{Q D}$ reaches its initial maximum value 1 . A pure state undergoing unitary evolution is highly coherent: it does not lose its coherence, i.e. off-diagonal coherences never vanish. $\delta_{Q D}=0$ when the quantum 
coherence is completely lost. So, when $\delta_{Q D}=1$ there is no QD and only if $\delta_{Q D}<1$, there is a significant degree of QD, when the magnitude of the elements of the density matrix in the position basis are peaked preferentially along the diagonal $q=q^{\prime}$. When $\delta_{Q D} \ll 1$, we have a strong QD.

b) Degree of classical correlations $(C C)$

In defining the degree of CC, the form of the Wigner function is essential, but not its position around $\sigma_{q}(t)$ and $\sigma_{p}(t)$. Consequently, for simplicity we consider zero values for the initial expectations values of the coordinate and momentum and the expression (26) of the Wigner function becomes

$$
W(q, p, t)=\frac{1}{2 \pi \hbar} \sqrt{\frac{\alpha}{\gamma}} \exp \left[-\frac{(\hbar \beta q-p)^{2}}{4 \hbar^{2} \gamma}-\alpha q^{2}\right] .
$$

A ridge of the Wigner function (33) in phase space is at $p=\hbar \beta q$, showing the correlation between $q$ and $p$. As a measure of the degree of CC we take the relative sharpness of this peak in the phase space determined from the dispersion $\hbar \sqrt{2 \gamma}$ in $p$ in Eq. (33) and the magnitude of the average of $p\left(p_{0}=\hbar \beta q\right)$ [4]:

$$
\delta_{C C}=\frac{2 \sqrt{\alpha \gamma}}{|\beta|}
$$

where we identified $q$ as the dispersion $1 / \sqrt{2 \alpha}$ of $q . \delta_{C C}$ is a good measure of the "squeezing" of the Wigner function in phase space [4]: in the state (33), more "squeezed" is the Wigner function, more strongly established are CC. In the coordinates $\hbar \beta q-p$ and $\hbar \beta q$ (these quantities have the same dimension), $2 \hbar \sqrt{\gamma}$ and $\hbar|\beta| / \sqrt{\alpha}$ are the lengths of the shorter and longer semi-axes of the $1 \sigma$ contour in phase space and their ratio gives $\delta_{C C}$. Similarly, in coordinates $\hbar \beta q-p$ and $q, 2 \hbar \sqrt{\gamma}$ and $1 / \sqrt{\alpha}$ are the lengths of the shorter and longer semi-axes of the $1 \sigma$ contour and their product gives the area of the $1 \sigma$ ellipse. We see from Eq. (27) that $\delta_{Q D}$ is inversely proportional to this area. Besides this geometric interpretation, $\delta_{Q D}$ is also connected with the linear entropy [34, 35].

For our case, we obtain

$$
\delta_{C C}(t)=\frac{\sqrt{\sigma(t)}}{\left|\sigma_{p q}(t)\right|}
$$

where $\sigma(t)$ is given by Eq. (29) and $\sigma_{p q}(t)$ can be calculated using formulas given in Refs. [27, 28]:

$$
\begin{array}{r}
\sigma_{p q}(t)=\frac{\hbar}{4 \Omega^{2}} e^{-2 \lambda t}\left\{\left[\mu \omega\left(2 \operatorname{coth} \epsilon-\delta-\frac{1}{\delta\left(1-r^{2}\right)}\right)-\frac{2 \omega^{2} r}{\sqrt{1-r^{2}}}\right] \cos (2 \Omega t)\right. \\
\left.+\omega \Omega\left(\delta-\frac{1}{\delta\left(1-r^{2}\right)}\right) \sin (2 \Omega t)+\mu \omega\left(\delta+\frac{1}{\delta\left(1-r^{2}\right)}-2 \operatorname{coth} \epsilon\right)+\frac{2 \mu^{2} r}{\sqrt{1-r^{2}}}\right\} .
\end{array}
$$




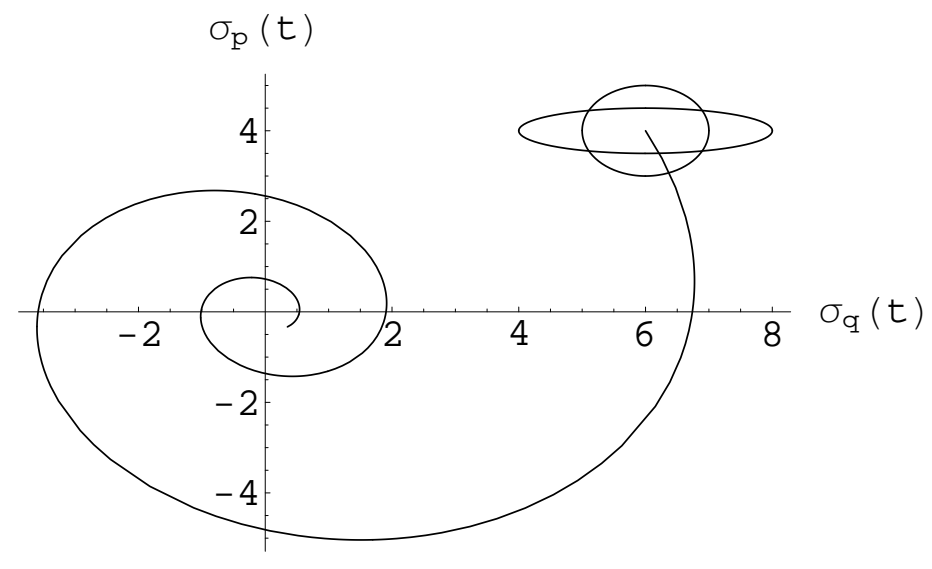

Figure 1: Trajectory in phase space given by the expectation values of coordinate $\sigma_{q}(t)$ and momentum $\sigma_{p}(t)$ for time $t \in[0,14]$ with initial coordinate $\sigma_{q}(0)=6$ and momentum $\sigma_{p}(0)=4$ and $1 \sigma$ contours of the Wigner function, corresponding to an initial coherent state $(\delta=1)$ and a squeezed state $(\delta=4)$, for $\lambda=0.2$ and $\mu=0.1$. In all figures we use the system of units $m=\omega=\hbar=1$.

When $\delta_{C C}$ is of order of unity, we have a significant degree of classical correlations. The condition of strong $\mathrm{CC}$ is $\delta_{C C} \ll 1$, which assures a very sharp peak in phase space. Since $\sigma_{p q}(\infty)=0$, in the case of an asymptotic Gibbs state, we get $\delta_{C C}(\infty) \rightarrow \infty$, so that our expression shows no CC at $t \rightarrow \infty$.

c) Discussion with Gaussian density matrix and Wigner function

We have seen that if the initial wave function is Gaussian, then the density matrix (18) and the Wigner function (21) remain Gaussian for all times (with time-dependent parameters which determine their amplitude and spread) and centered along the trajectory given by Eqs. (9) and (10), which are the solutions $\sigma_{q}(t)$ and $\sigma_{p}(t)$ of the dissipative equations of motion (7) and (18). This trajectory is exactly classical for $\lambda=\mu$ and only approximately classical for not large $\lambda-\mu$. In Fig. 1 there are represented the trajectory in phase space and two examples of the $1 \sigma$ contour of the initial Wigner function, corresponding to an initial coherent state $(\delta=1)$ and a squeezed state $(\delta=4)$. In general, the $1 \sigma$ contour is defined by the ellipse

$$
\frac{1}{2 \sigma(t)}\left[\sigma_{p p}(t)\left(q-\sigma_{q}(t)\right)^{2}+\sigma_{q q}(t)\left(p-\sigma_{p}(t)\right)^{2}-2 \sigma_{p q}(t)\left(q-\sigma_{q}(t)\right)\left(p-\sigma_{p}(t)\right)\right]=1 .
$$

In Fig. 1 the center of the ellipses is the point given by the initial expectation values $\sigma_{q}(0)$ and $\sigma_{q}(0)$.

To illustrate the dependence on the temperature and time of the degree of QD and the degree of CC, we represent them in Fig. 2. We can see that the degree of 
QD has an evolution which shows that in general QD increases with time and temperature. We can also see that the degree of CC has a more complicated evolution, but the general tendency is that CC are less and less strong with increasing time and temperature. $\delta_{Q D}<1$ for non-zero temperature and $\delta_{C C}$ is of the order of unity for a long enough interval of time, so that we can say that the considered system interacting with the thermal bath manifests both $\mathrm{QD}$ and $\mathrm{CC}$ and a true quantum to classical transition takes place. Dissipation promotes quantum coherences, whereas fluctuation (diffusion) reduces coherences and promotes QD. The balance of dissipation and fluctuation determines the final equilibrium value of $\delta_{Q D}$. The quantum system starts as a pure state, with a Wigner function well localized in phase space (Gaussian form). This state evolves approximately following the classical trajectory (Liouville flow) in phase space and becomes a quantum mixed state during the irreversible process of QD.

The squeezing and the correlation of the initial state play also a role in the degree of $\mathrm{QD}$ and CC. When the squeezing parameter $\delta$ is increasing, both $\delta_{Q D}$ and $\delta_{C C}$ are decreasing, therefore the squeezing favorizes both QD and CC. Likewise, the increasing of the correlation coefficient $r$ leads to the decreasing of the degree of $\mathrm{QD}$, so that when we have a larger initial correlation, then QD is stronger. At the same time, the variation of the correlation coefficient has a small influence on the general pulsatory behaviour of the degree of CC. We also remark from Eq. (31) that the asymptotic value of the degree of QD does not depend on the initial squeezing and correlation, it depends on temperature only.

In Figs. 3 and 4 we represent the density matrix in coordinate representation (18) and the Wigner function (21) at the initial and final moments of time. The values of the density matrix along the diagonal $q=q^{\prime}$ represent the probability of finding the system in this position, while the off-diagonal values represent the correlations in the density matrix between the points $q$ and $q^{\prime}$. The asymptotic Wigner distribution has an axial symmetry, reflecting quantum equipartition. For simplicity, in Figs. 3 and 4 we consider zero values for the initial expectations values of coordinate and momentum, so that both the density matrix and Wigner function are centered in origin. Of course, as we stated earlier, for non-zero initial expectations values of coordinate and momentum, the density matrix (18) and the Wigner function (21) are centered along the trajectory given by Eqs. (9) and (10), like in Fig. 1.

From expressions (27) and (34) we notice that the key parameter which describes $\mathrm{QD}$ and $\mathrm{CC}$ is $\gamma$. This coefficient determines the spread of the Wigner function (26) around the path in phase space and measures the contribution of non-diagonal terms 

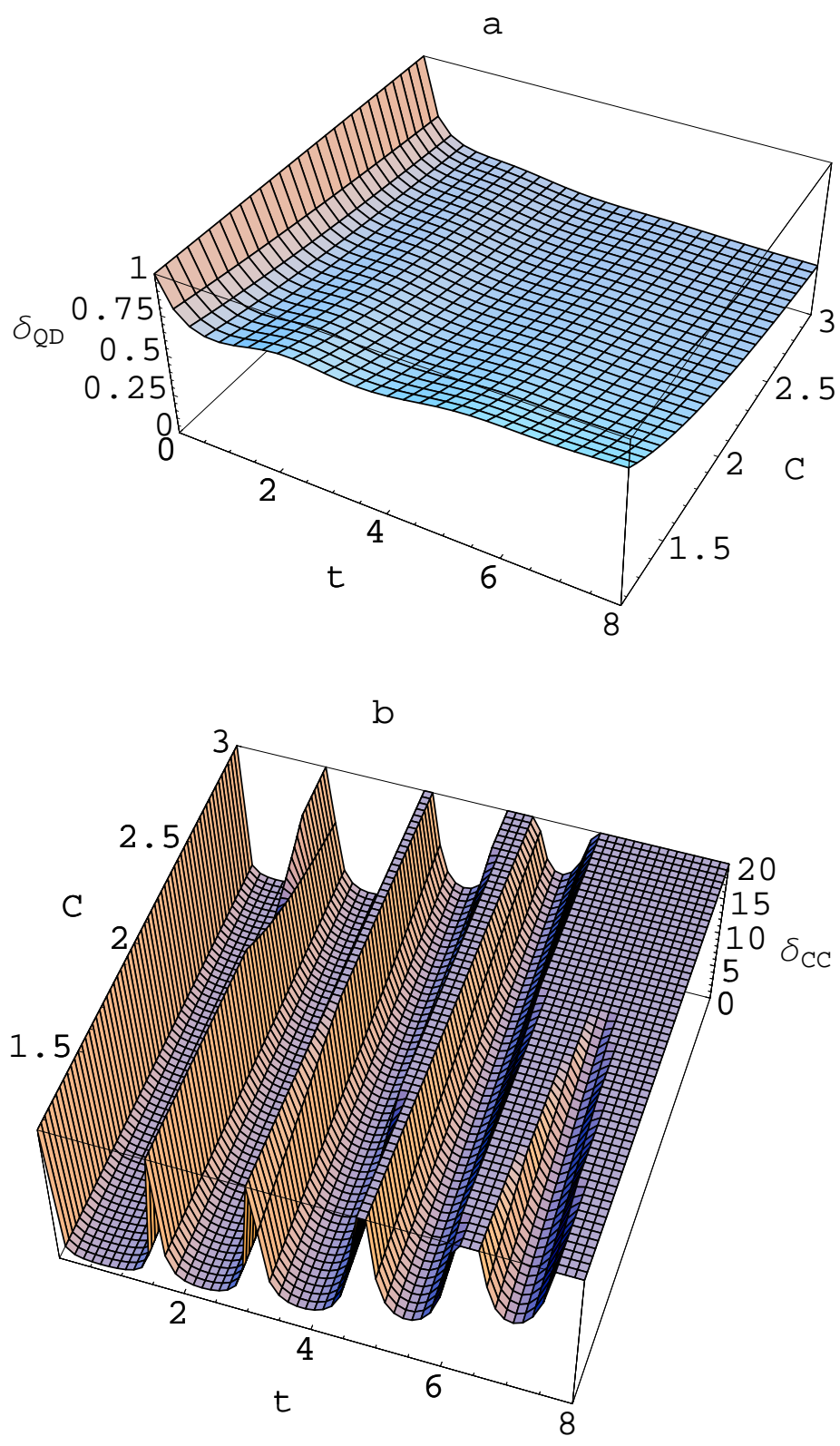

Figure 2: a: Degree of quantum decoherence $\delta_{Q D}$ and b: degree of classical correlations $\delta_{C C}$ as functions on temperature $T$ (through $C \equiv \operatorname{coth} \frac{\hbar \omega}{2 k T}$ ) and time $t$ for $\lambda=0.2, \mu=$ $0.1, \delta=4, r=0$. 

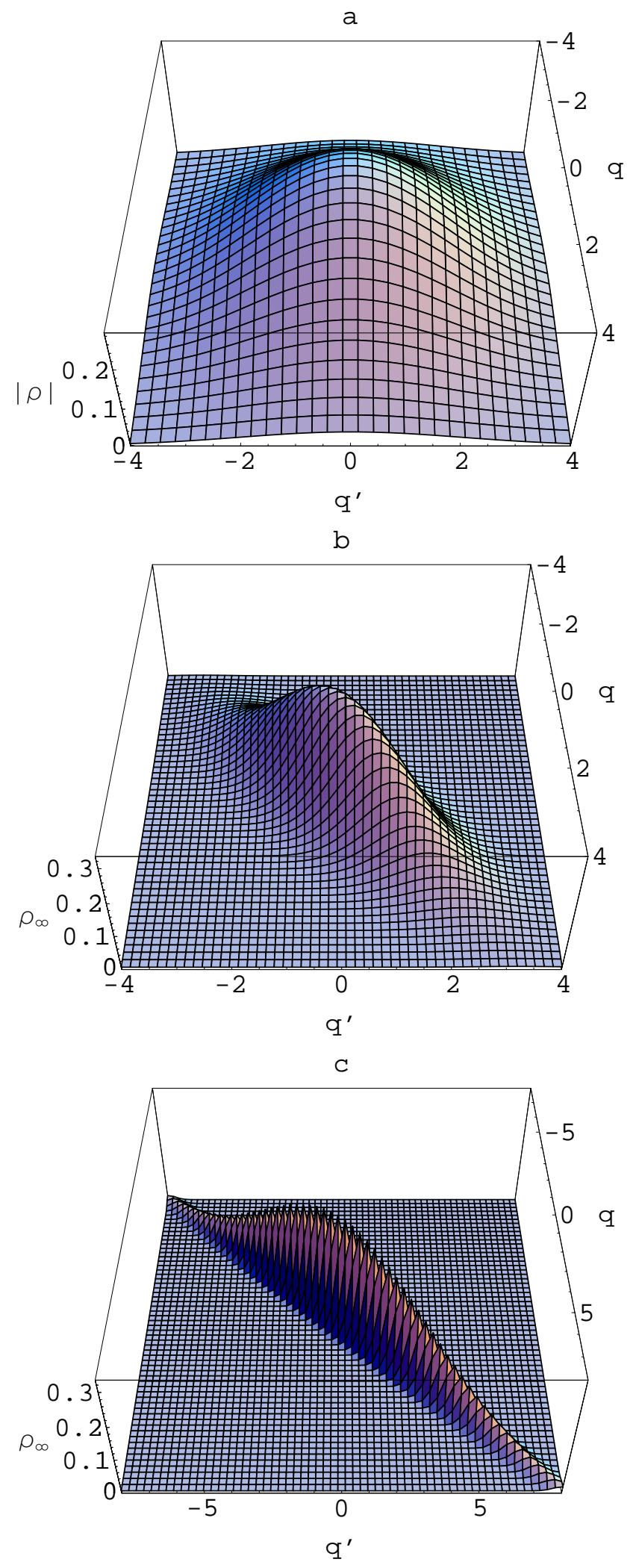

Figure 3: Density matrix $\rho$ in coordinate representation for $\lambda=0.2, \mu=0.1, \delta=4, r=$ 0 ; a: $|\rho|$ at the initial time $t=0$; b: $\rho_{\infty}$ at time $t \rightarrow \infty$ for $C=3$; c: $\rho_{\infty}$ for $C=20$. 

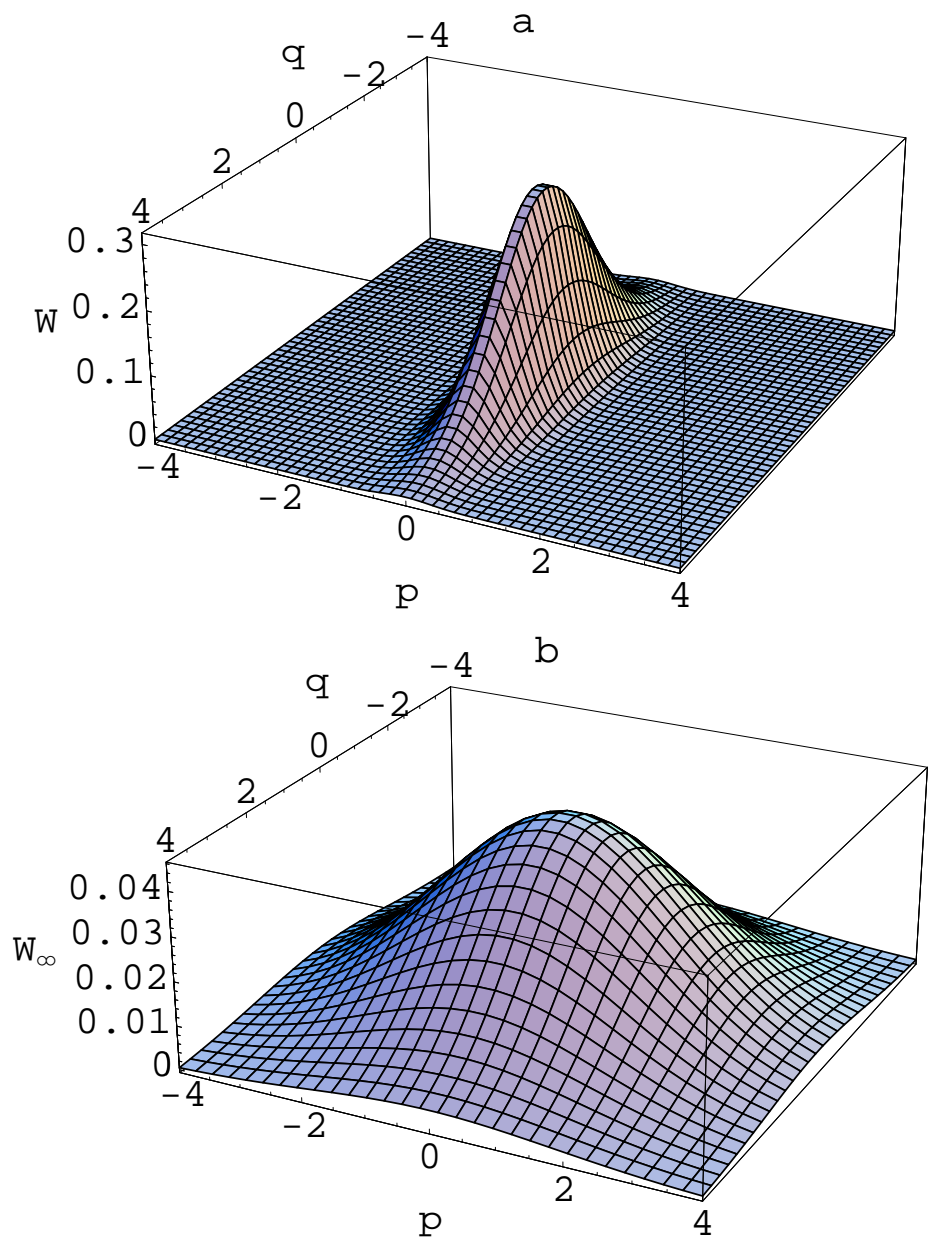

Figure 4: Wigner function $W$ for $\lambda=0.2, \mu=0.1, \delta=4, r=0$ and $C=3$; a: $t=0$; b: $t \rightarrow \infty$. 
in the density matrix (24). Therefore, when decoherence increases, the correlations between the canonical variables of coordinate and momentum decrease. The extreme limit of $\mathrm{QD}(\gamma \rightarrow \infty)$ is incompatible with $\mathrm{CC}$ and that of $\mathrm{CC}(\gamma \rightarrow 0)$ is incompatible with QD. Their simultaneous realization is not a trivial task: QD requires interaction with an environment, which inevitably suppresses $\mathrm{CC}$ and produces fluctuations in the evolution of the system, whereas classical predictability requires these fluctuations to be small. Therefore the existence of the environment is crucial for the quantum to classical transition and, consequently, classicality is an emergent property of an open quantum system. The shown figures confirm the presence of the relative competition which appears between QD and existence of CC, since decoherence (diagonalizing or the decreasing of the width of the density matrix) implies a spreading of the Wigner distribution function (which is the Fourier transform of the density matrix) along the trajectory in phase space, whereas CC require the existence of sharp peaks in the Wigner function. Although there exists this competition, there is a broad compromise regime in which QD and CC can hold well simultaneously. If the density matrix is not diagonal, but the Wigner function becomes peaked along the classical trajectory for long times, we do not have, strictly speaking, a classical limit, but only a classical behaviour and CC. We regard classical behaviour as a quantum behaviour in which there exists correlations between coordinate and momentum or when coherences are negligible, even without having $\mathrm{CC}$.

We can assert that in the considered case classicality is a temporary phenomenon, which takes place only at some stages of the dynamical evolution, during a definite interval of time. Due to the dissipative nature of evolution, the approximately deterministic evolution is no more valid for very large times, when the localization of the system is affected by the spreading of the wave packet and of the Wigner distribution function.

In the case of a closed harmonic oscillator (zero-damping limit, $\lambda=0$ ), $\delta_{Q D}=1$ and the QD phenomenon does not take place. At the same time we obtain (if $r=0$ )

$$
\delta_{C C}(t)=\frac{2}{\left|\left(\delta-\frac{1}{\delta}\right) \sin (2 \omega t)\right|} .
$$

Consequently, for an initial coherent state $(\delta=1)$, we see that $\delta_{C C}=\infty$ for any time, so that there are no CC. If the initial state is squeezed $(\delta \neq 1)$, then the system manifests $\mathrm{CC}$ and it has a classical behaviour. 


\section{Decoherence time scale and transition from quan- tum mechanics to classical statistical mechanics}

Diffusion in momentum, which generates the decoherence in coordinate $q$, occurs at the rate set by $D_{p p}$. In the macroscopic limit, when $\hbar$ is small compared to other quantities with dimensions of action, such as $\sqrt{D_{p p}<\left(q-q^{\prime}\right)^{2}>}$, the term in Eq. (16) containing $D_{p p} / \hbar^{2}$ dominates and induces the following evolution of the density matrix:

$$
\frac{\partial \rho}{\partial t}=-\frac{D_{p p}}{\hbar^{2}}\left(q-q^{\prime}\right)^{2} \rho
$$

Thus the density matrix loses off-diagonal terms in position representation:

$$
<q|\rho(t)| q^{\prime}>=<q|\rho(0)| q^{\prime}>\exp \left[-\frac{D_{p p}}{\hbar^{2}}\left(q-q^{\prime}\right)^{2} t\right]
$$

while the diagonal $\left(q=q^{\prime}\right)$ ones remain untouched. Quantum coherences decay exponentially at a rate given by

$$
\frac{D_{p p}}{\hbar^{2}}\left(q-q^{\prime}\right)^{2}
$$

so that the decoherence time scale is of the order of

$$
\frac{\hbar^{2}}{D_{p p}\left(q-q^{\prime}\right)^{2}} \text {. }
$$

In the case of a thermal bath, we obtain (see Eq. (5) )

$$
t_{\text {deco }}=\frac{2 \hbar}{(\lambda+\mu) m \omega \sigma_{q q}(0) \operatorname{coth} \epsilon}
$$

where we have taken $\left(q-q^{\prime}\right)^{2}$ of the order of the initial dispersion in coordinate $\sigma_{q q}(0)$.

In order to obtain a more precise expression of the decoherence time, we consider the coefficient $\gamma(\underline{25})$, which measures the contribution of non-diagonal terms in the density matrix (24). For short times $(\lambda t \ll 1, \Omega t \ll 1)$, we have:

$\gamma(t)=-\frac{m \omega}{4 \hbar \delta}\left\{1+2\left[\lambda\left(\delta+\frac{r^{2}}{\delta\left(1-r^{2}\right)}\right) \operatorname{coth} \epsilon+\mu\left(\delta-\frac{r^{2}}{\delta\left(1-r^{2}\right)}\right) \operatorname{coth} \epsilon-\lambda-\mu-\frac{\omega r}{\delta \sqrt{1-r^{2}}}\right] t\right\} .($

From here we obtain that quantum coherences in the density matrix decay exponentially at a rate given by

$$
2\left[\lambda\left(\delta+\frac{r^{2}}{\delta\left(1-r^{2}\right)}\right) \operatorname{coth} \epsilon+\mu\left(\delta-\frac{r^{2}}{\delta\left(1-r^{2}\right)}\right) \operatorname{coth} \epsilon-\lambda-\mu-\frac{\omega r}{\delta \sqrt{1-r^{2}}}\right]
$$


and then the decoherence time scale is

$$
t_{\text {deco }}=\frac{1}{2\left[\lambda\left(\delta+\frac{r^{2}}{\delta\left(1-r^{2}\right)}\right) \operatorname{coth} \epsilon+\mu\left(\delta-\frac{r^{2}}{\delta\left(1-r^{2}\right)}\right) \operatorname{coth} \epsilon-\lambda-\mu-\frac{\omega r}{\delta \sqrt{1-r^{2}}}\right]} .
$$

The decoherence time depends on the temperature $T$ and the coupling $\lambda$ (dissipation coefficient) between the system and environment (through the diffusion coefficient $D_{p p}$ ), on the squeezing parameter $\delta$ that measures the spread in the initial Gaussian packet and on the initial correlation coefficient $r$. We notice that the decoherence time is decreasing with increasing dissipation, temperature and squeezing.

For $r=0$ we obtain:

$$
t_{\text {deco }}=\frac{1}{2(\lambda+\mu)(\delta \operatorname{coth} \epsilon-1)}
$$

and at temperature $T=0$ (then we have to take $\mu=0$ ), this becomes

$$
t_{\text {deco }}=\frac{1}{2 \lambda(\delta-1)} .
$$

We see that when the initial state is the usual coherent state $(\delta=1)$, then the decoherence time tends to infinity. This corresponds to the fact that for $T=0$ and $\delta=1$ the coefficient $\gamma$ is constant in time, so that the decoherence process does not occur in this case.

At high temperature, introducing the notation

$$
\tau \equiv \frac{2 k T}{\hbar \omega} \equiv \frac{1}{\epsilon}
$$

expression (46) becomes

$$
t_{\text {deco }}=\frac{1}{2\left[\lambda\left(\delta+\frac{r^{2}}{\delta\left(1-r^{2}\right)}\right)+\mu\left(\delta-\frac{r^{2}}{\delta\left(1-r^{2}\right)}\right)\right] \tau} .
$$

If, in addition $r=0$, then we obtain

$$
t_{\text {deco }}=\frac{\hbar \omega}{4(\lambda+\mu) \delta k T} .
$$

In Ref. [30] we studied the behaviour of the generalized uncertainty function $\sigma(t)$ (29). For short times we obtained

$$
\sigma(t)=\frac{\hbar^{2}}{4}\left\{1+2\left[\lambda\left(\delta+\frac{1}{\delta\left(1-r^{2}\right)}\right) \operatorname{coth} \epsilon+\mu\left(\delta-\frac{1}{\delta\left(1-r^{2}\right)}\right) \operatorname{coth} \epsilon-2 \lambda\right] t\right\} .
$$

This expression shows explicitly the contribution for small time of: (i) uncertainty that is intrinsic to quantum mechanics, expressed through the Heisenberg uncertainty principle 
and (ii) uncertainty due to the coupling to the thermal environment, which has two components, dissipation and diffusion (this last component is responsible for the process of decoherence). From Eq. (52) we can determine the time $t_{d}$ when statistical (thermal) fluctuations become comparable with quantum fluctuations. At high temperature we obtain

$$
t_{d}=\frac{1}{2 \tau\left[\lambda\left(\delta+\frac{1}{\delta\left(1-r^{2}\right)}\right)+\mu\left(\delta-\frac{1}{\delta\left(1-r^{2}\right)}\right)\right]} .
$$

By statistical (thermal) fluctuations we mean the fluctuations produced by diffusion, that arise in the generalized uncertainty function $\sigma(t)$ from the coupling of the harmonic oscillator to the thermal bath at arbitrary temperature $T$, even at $T=0$ (when the diffusion coefficient still has a non-zero value). By quantum fluctuations we mean fluctuations of the quantum harmonic oscillator at zero coupling with the thermal bath.

As expected, we can see that the decoherence time $t_{\text {deco }}$ has the same scale as the time $t_{d}$ after which statistical fluctuations become comparable with quantum fluctuations. The values of $t_{\text {deco }}$ and $t_{d}$ become closer with increasing temperature and squeezing.

When $t \gg t_{r e l}$, where $t_{r e l} \approx \lambda^{-1}$ is the relaxation time, which governs the rate of energy dissipation, the particle reaches equilibrium with the environment. Indeed, the uncertainty function $\sigma(t)(\underline{29})$ is insensitive to $\lambda, \mu, \delta$ and $r$ and approaches

$$
\sigma^{B E}=\frac{\hbar^{2}}{4} \operatorname{coth}^{2} \epsilon .
$$

This is the Bose-Einstein relation for a system of bosons in equilibrium at temperature $T$, obtained also in quantum Brownian models for the weak coupling at arbitrary temperature. In the case of $T=0$ we approach the limit of pure quantum fluctuations,

$$
\sigma_{0}=\frac{\hbar^{2}}{4},
$$

which is the quantum Heisenberg relation. At high temperatures $T(T \gg \hbar \omega / k)$ we obtain the limit of pure thermal fluctuations,

$$
\sigma^{M B}=\left(\frac{k T}{\omega}\right)^{2}
$$

which is a Maxwell-Boltzmann distribution for a system approaching a classical limit.

For all macroscopic bodies the dissipation term becomes important much later after the decoherence term has already dominated and diminished the off-diagonal terms. If 
we compare the time scales of these two terms (decoherence rate (41) and relaxation rate) we get for high temperatures (for $\mu=0$ )

$$
\frac{\text { Decoherence rate }}{\text { Relaxation rate }}=\frac{\frac{D_{p p}(\Delta q)^{2}}{\hbar^{2}}}{\lambda}=\frac{m \omega}{2 \hbar}\left(q-q^{\prime}\right)^{2} \operatorname{coth} \frac{\hbar \omega}{2 k T} \approx \frac{m k T}{\hbar^{2}}\left(q-q^{\prime}\right)^{2} \text {. }
$$

In most typical situations this is a huge number. For example, for a mass of $1 \mathrm{~g}$ at room temperature $(T=300 \mathrm{~K})$ and for a separation of $q-q^{\prime}=1 \mathrm{~cm}$, the decoherence time scale $t_{\text {deco }}$ is approximately $10^{40}$ times shorter than the relaxation time $t_{r e l}$, so that in the macroscopic domain QD occurs very much faster than relaxation. We remark also that $t_{\text {deco }}$ can be of the order of $t_{r e l}$ for sufficiently low temperatures and small wave packet spread (small squeezing coefficient). At the same time we have to remind that Lindblad theory is obtained in the Markovian approximation, when the characteristic time scales of the considered processes are larger than the characteristic time scales of the thermal bath. When the decoherence time scale is much shorter than the relaxation time and at the same time the decoherence becomes faster even than the environment time scales, then Markovian approximation is no more valid and the use of non-Markovian quantum diffusion models becomes preferable.

We have seen that a necessary condition for a system to behave classically is the QD process. The time scale after which the system has a classical behaviour, with an evolution described by a classical probability distribution, is determined by the decoherence time $t_{\text {deco }}$, when the density matrix becomes approximately diagonal very rapidly during the system-environment interaction. On the other hand, one often regards the regime where statistical fluctuations begin to surpass quantum fluctuations as the transition point from quantum to classical statistical mechanics and identifies the high temperature regime of a system as the classical regime. Above it was shown that these two criteria of classicality are equivalent: the time when the quantum system decoheres is comparable with the time when statistical fluctuations overtake quantum fluctuations $\left(t_{\text {deco }} \approx t_{d}\right)$. This result is a new confirmation of the previous similar results [36, 37, 38]. However the regime after statistical fluctuations dominate should not be called classical. After the decoherence time, although the system is describable in terms of probabilities, it can not yet be regarded as classical because of the spin-statistics effects. It has to be described by non-equilibrium quantum statistical mechanics. After the relaxation time the system is correctly treated by the equilibrium quantum statistical mechanics, and only at a sufficiently high temperature, when the spin (Fermi-Dirac or Bose-Einstein) statistics can be represented by the Maxwell-Boltzmann distribution function, it can be considered in a classical regime [30, 37, 38] (see Eqs. (54), (56)). 


\section{Summary and concluding remarks}

We would like to remark that there are no precise quantitative criteria in literature for classicality and at the same time there exist some ambiguity and even unclarities concerning the characteristics of the quantum to classical transition. In the present paper we have studied QD and CC with the Markovian equation of Lindblad in order to understand the transition from quantum to classical mechanics for a system consisting of an one-dimensional harmonic oscillator in interaction with a thermal bath in the framework of the theory of open quantum systems based on quantum dynamical semigroups. Our results may be summarized as follows.

(1) Using the criterion of QD for the considered model, we have shown that QD in general increases with time and temperature. For large temperatures, QD is strong and the degree of mixedness is high, while for zero temperature the asymptotic final state is pure. With increasing squeezing parameter and initial correlation, QD becomes stronger, but the asymptotic value of the degree of QD does not depend on the initial squeezing and correlation, it depends on temperature only.

(2) Using the criterion of CC, we have shown that the general tendency is that $\mathrm{CC}$ are less and less strong with increasing time and temperature. For a long enough interval of time we have a significant degree of $\mathrm{CC}$, but at $t \rightarrow \infty$ there are no $\mathrm{CC}$ in the case of an asymptotic Gibbs state. With increasing squeezing parameter, CC become stronger, but the variation of the correlation coefficient has a small influence on the behaviour of the degree of CC.

(3) During a finite interval of time the system interacting with the thermal bath manifests simultaneously both QD and CC, so that a true quantum to classical transition takes place and the system recovers classicality in a significant measure. CC are expressed by the fact that the Wigner function has a peak which follows (exactly for $\lambda=\mu$ and approximately for $\lambda \neq \mu$ ) the classical trajectory in phase space and QD is expressed by the loss of quantum coherence in the case of a thermal bath at finite temperature. For an initial Gaussian quantum state, Wigner function is positive for all times, so that it represents a true classical probability distribution in phase space.

(4) The expressions of the degree of QD and CC confirm the relative competition which appears between QD and existence of $\mathrm{CC}$, since decoherence implies a spreading of the Wigner distribution function along the trajectory in phase space, whereas CC require the existence of sharp peaks in the Wigner function. However, there exists a broad compromise regime in which $\mathrm{QD}$ and $\mathrm{CC}$ can hold well simultaneously. Consequently, classicality is a temporary phenomenon, which takes place only at some stages of the 
dynamical evolution of the system, during a definite interval of time.

(5) We determined the general expression of the decoherence time, which shows that it is decreasing with increasing dissipation, temperature and squeezing. We have also shown that the decoherence time has the same scale as the time after which statistical fluctuations become comparable with quantum fluctuations, as expected, and the values of these scales become closer with increasing temperature and squeezing. After the decoherence time, the decohered system is not necessarily in a classical regime. There exists a quantum statistical regime in between. For the considered open system at a finite temperature, the uncertainty relation (54) holds, which interpolates between the Heisenberg relation at zero temperature (55) and the high temperature classical statistical relation (56). Only at a sufficiently high temperature, when the spin statistics can be represented by the Maxwell-Boltzmann distribution, the system can be considered in a classical regime.

The study of classicality using QD and CC leads to a deeper understanding of the quantum origins of the classical world. As a result of the progress made in the last two decades, the quantum to classical transition has become a subject of experimental investigations, while previously it was mostly a domain of theory [2, 3]. The issue of quantum to classical transition points to the necessity of a better understanding of open quantum systems. The Lindblad theory provides a self-consistent treatment of damping as a general extension of quantum mechanics to open systems and gives the possibility to extend the model of quantum Brownian motion. The obtained results in the framework of the Lindblad theory are a useful basis for the description of the connection between uncertainty, decoherence and correlations (entanglement) of open quantum systems with their environment.

\section{Acknowledgments}

Financial support and hospitality at the Institute of Theoretical Physics in Giessen (Germany) during the stay of one (A. I.) of the authors are gratefully acknowledged. The authors would also like to thank the referee for useful criticism and recommendations.

\section{References}

[1] E. Joos, H. D. Zeh, C. Kiefer, D. Giulini, J. Kupsch and I. O. Stamatescu, Decoherence and the Appearance of a Classical World in Quantum Theory, 2nd Edn (Springer, Berlin, 2003) 
[2] J. P. Paz and W. H. Zurek, in Coherent Atomic Matter Waves, Les Houches Session LXXII, ed. by R. Kaiser, C. Westbrook and F. David (Springer, Berlin, 2001), p. 533

[3] W. H. Zurek, Rev. Mod. Phys. 75, 715 (2003)

[4] M. Morikawa, Phys. Rev. D 42, 2929 (1990)

[5] S. Habib and R. Laflamme, Phys. Rev. D 42, 4056 (1990)

[6] R. Alicki, Open Sys. and Information Dyn. 11, 53 (2004)

[7] H. D. Zeh, Found. Phys. 1, 69 (1970); 3, 109 (1973)

[8] W. H. Zurek, Phys. Rev. D 24, 1516 (1981) W. H. Zurek, Phys. Rev. D 26, 1862 (1982)

[9] E. Joos, Phys. Rev. D 29, 1626 (1984)

[10] E. Joos and H. D. Zeh, Z. Phys. B 59, 223 (1985)

[11] A. O. Caldeira and A. J. Leggett, Phys. Rev. A 31, 1059 (1985)

[12] W. G. Unruh and W. H. Zurek, Phys. Rev. D 40, 1071 (1989)

[13] B. L. Hu, J. P. Paz and Y. Zhang, Phys. Rev. D 45, 2843 (1992)

[14] J. P. Paz, S. Habib and W. H. Zurek, Phys. Rev. D 47, 488 (1993)

[15] W. H. Zurek, Phys. Today 44, No. 10, 36 (1991); 46, No. 12, 81 (1993); Prog. Theor. Phys. 89, 281 (1993); Physical Origins of Time Asymmetry, ed. by J. Halliwell, J. Perez-Mercader and W. Zurek (Cambridge University Press, Cambridge, 1994)

[16] M. Brune, E. Hagley, J. Dreyer, X. Maître, A. Maali, C. Wunderlich, J.-M. Raimond and S. Haroche, Phys. Rev. Lett. 77, 4887 (1996)

[17] C. C. Cheng and M. G. Raymer, Phys. Rev. Lett. 82, 4807 (1999)

[18] H. Ammann, R. Gray, I. Shvarchuck and N. Christensen, Phys. Rev. Lett. 80, 4111 (1998)

[19] B. G. Klappauf, W. H. Oskay, D. A. Steck and M. G. Raizen, Phys. Rev. Lett. 81, 1203 (1998); Erratum in Phys. Rev. Lett. 82, 241 (1999) 
[20] Q. A. Turchette, C. J. Myatt, B. E. King, C. A. Sackett, D. Kielpinski, W. H. Itano et. al, Phys. Rev. A 62, 053807 (2000)

[21] D. A. Kokorowski, A. D. Cronin, T. D. Roberts, D. E. Pritchard, Phys. Rev. Lett. 86, 2191 (2001)

[22] M. A. Nielsen and I. L. Chuang, Quantum Computation and Quantum Information (Cambridge Univ. Press, Cambridge, 2000)

[23] E. B. Davies, Quantum Theory of Open Systems (Academic Press, New York, 1976)

[24] G. Lindblad, Commun. Math. Phys. 48, 119 (1976)

[25] H. Spohn, Rev. Mod. Phys. 52, 569 (1980)

[26] G. Lindblad, Rep. Math. Phys. 10, 393 (1976)

[27] A. Sandulescu and H. Scutaru, Ann. Phys. (N.Y.) 173, 277 (1987)

[28] A. Isar, A. Sandulescu, H. Scutaru, E. Stefanescu and W. Scheid, Int. J. Mod. Phys. E 3, 635 (1994)

[29] V. V. Dodonov, E. V. Kurmyshev and V. I. Man'ko, Phys. Lett. A 79, 150 (1980)

[30] A. Isar and W. Scheid, Phys. Rev. A 66, 042117 (2002)

[31] A. Isar, W. Scheid and A. Sandulescu, J. Math. Phys. 32, 2128 (1991)

[32] A. Isar, Helv. Phys. Acta 67, 436 (1994)

[33] A. Isar, A. Sandulescu and W. Scheid, Int. J. Mod. Phys. B 10, 2767 (1996)

[34] A. Isar, Fortschr. Phys. 47, 855 (1999)

[35] A. Isar, A. Sandulescu and W. Scheid, Phys. Rev. E 60, 6371 (1999)

[36] C. Anastopoulos and J. J. Halliwell, Phys. Rev. D 51, 6870 (1995)

[37] B. L. Hu and Y. Zhang, Mod. Phys. Lett. A 8, 3575 (1993)

[38] B. L. Hu and Y. Zhang, Int. J. Mod. Phys. A 10, 4537 (1995) 